\title{
Supplementary materials to
}

\section{Intra-regional transport of black carbon between North China Plain and Central China during winter haze episodes}

Huang Zheng ${ }^{1,2}$, Shaofei Kong ${ }^{1}$, Fangqi Wu ${ }^{1}$, Yi Chen ${ }^{1}$, Zhenzhen Niu ${ }^{1}$, Shurui Zheng ${ }^{1}$, Guowei Yang ${ }^{1}$, Liquan $\mathrm{Yao}^{2}$, Qin Yan ${ }^{1,2}$, Jian $\mathrm{Wu}^{1,2}$, Mingming Zheng ${ }^{2,3}$, Nan $\mathrm{Chen}^{3}$, Ke $\mathrm{Xu}^{3}$, Yingying Yan ${ }^{1}$, Dantong $\mathrm{Liu}^{4}$, Delong Zhao ${ }^{5}$, Tianliang Zhao ${ }^{6}$, Yongqing $\mathrm{Bai}^{7}$, Shuanglin $\mathrm{Li}^{1}$, and Shihua $\mathrm{Qi}^{2}$

${ }^{1}$ Department of Atmospheric Science, School of Environmental Sciences, China University of Geosciences, Wuhan, 430074, China

${ }^{2}$ Department of Environmental Science and Technology, School of Environmental Sciences, China University of Geosciences, Wuhan, 430074, China

${ }^{3}$ Hubei Provincial Environmental Monitoring Centre, Wuhan, 430072, China

${ }^{4}$ School of Earth, Atmospheric \& Environmental Sciences, University of Manchester, M139PL, UK

${ }^{5}$ Beijing Weather Modification Office, Beijing, 100089, China

${ }^{6}$ School of Atmospheric Physics, Nanjing University of Information Science and Technology, Nanjing, 210044, China

${ }^{7}$ Hubei Key Laboratory for Heavy Rain Monitoring and Warning Research, Institute of Heavy Rain, China Meteorological Administration, Wuhan, 430205, China

Correspondence to: Shaofei Kong (kongshaofei@cug.edu.cn) 


\section{Text S1 Description of CWT}

To distinguish the pollution levels of different potential regions, a concentration-weighted-trajectory (CWT) model was employed in this study. The CWT was calculated according to:

$C_{\mathrm{ij}}=\frac{1}{\sum_{l=1}^{M} \tau_{i j l}} \sum_{l=1}^{N} c_{l} \tau_{i j l}$

where $C_{i j}$ represents the average weight concentrations in the grid cell $(\mathrm{i}, \mathrm{j}) ; C_{l}$ is the measured BC concentration observed on the arrival of trajectory $l ; \tau_{i j l}$ is the number of trajectory end points in the grid cell $(\mathrm{i}, \mathrm{j})$ associated with the $C_{l}$ sample.

In order to consider only air parcels with good representativeness, a weighing function was added to the calculation to down weight cells associated with low values of $n$. The default weighting function was used as below:

$W=\left\{\begin{array}{rr}1.00 & \text { for } \log (n+1) \geq 0.85 * \max _{\log (n+1)} \\ 0.725 & \text { for } 0.60 * \max _{\log (n+1)}>\log (n+1) \geq 0.85 * \max _{\log (n+1)} \\ 0.475 & \text { for } 0.35 * \max _{\log (n+1)}>\log (n+1) \geq 0.60 * \max _{\log (n+1)} \\ 0.175 & \text { for } \log (n+1)<0.35 * \max _{\log (n+1)}\end{array}\right.$ 
Table S1 Reported BC levels in China and around the world.

\begin{tabular}{|c|c|c|c|c|c|c|c|}
\hline Station & Site type & Location $\left({ }^{\circ} \mathrm{N}\right),\left({ }^{\circ} \mathrm{E}\right)$ & Inlet & Instrument & Sampling period & $\mathrm{BC}\left(\mu \mathrm{g} \mathrm{m}^{-3}\right)$ & References \\
\hline Hongan & Rural & $31.24,114.58$ & $\mathrm{PM}_{2.5}$ & AE33 & 2018.01 & $5.54 \pm 2.59$ & This study \\
\hline Luohe & Suburban & $33.57,114.05$ & $\mathrm{PM}_{2.5}$ & AE33 & 2018.01 & $8.48 \pm 4.83$ & \\
\hline Suixian & Rural & $31.88,113.28$ & - & AE51 & 2018.01 & $4.47 \pm 2.90$ & \\
\hline Wuhan & Urban & $30.53,114.39$ & $\mathrm{PM}_{2.5}$ & AE31 & 2018.01 & $3.91 \pm 1.86$ & \\
\hline Xiangyang & Suburban & $32.02,112.17$ & - & AE51 & 2018.01 & $7.35 \pm 3.45$ & \\
\hline \multicolumn{8}{|l|}{ YRD } \\
\hline Nanjing & Urban & $32.05,118.78$ & & & 2012 Annual & $4.16 \pm 2.63$ & (Zhuang et al., 2014) \\
\hline Shanghai & Urban & $31.23,121.53$ & & AE31 & 2007.04-2010.03 & 3.83 & (Wang et al., 2014a) \\
\hline Jiaxin & Suburban & $30.80,120.8$ & & $\mathrm{SP} 2$ & 2010.12 & 7.1 & (Huang et al., 2013) \\
\hline \multicolumn{8}{|l|}{ PRD } \\
\hline Maofeishan & Rural & $23.33,113.48$ & $\mathrm{PM}_{10}$ & AE31 & $2008.12-2009.01$ & 2.88 & \multirow[t]{5}{*}{ (Wu et al., 2013) } \\
\hline Nancuan & Suburban & $23.00,113,35$ & $\mathrm{PM}_{10}$ & AE31 & 2008.12-2009.01 & 7.68 & \\
\hline Panyu (PY) & Urban & $22.93,113.32$ & $\mathrm{PM}_{10}$ & AE31 & $2008.12-2009.01$ & 20.21 & \\
\hline Dongguan & Suburban & $22.97,113.73$ & $\mathrm{PM}_{10}$ & AE31 & $2008.12-2009.01$ & 10.11 & \\
\hline Xinken & Rural & $22.71,113.55$ & $\mathrm{PM}_{10}$ & AE31 & 2008.12-2009.01 & 12.61 & \\
\hline Hong Kong & Rural-costal & $22.22,114.25$ & $\mathrm{PM}_{2.5}$ & AE31 & $2012.02-2015.02$ & 1 & (Wang et al., 2017) \\
\hline Shenzhen & Urban & $22.60,113.97$ & & \multirow{2}{*}{$\mathrm{SP} 2$} & 2010.01-02 & $4.1 \pm 3.8$ & \multirow{2}{*}{ (Huang et al., 2012) } \\
\hline Shenzhen & Rural & $22.65,114.53$ & & & $2009.11-12$ & $2.6 \pm 1.0$ & \\
\hline \multicolumn{8}{|l|}{ NCP } \\
\hline Beijing & Suburban & $40.65,117.12$ & - & AE31 & 2003.04-2005.01 & $2.12 \pm 1.62$ & (Yan et al., 2008) \\
\hline Beijing & Urban & $39.93,116.30$ & $\mathrm{PM}_{2.5}$ & AE31 & $2010.01-2014.12$ & 3.67 & (Liu et al., 2016) \\
\hline Beijing & Urban & $39.97,116.37$ & & SP2 & 2013.01 & 5.5 & (Wu et al., 2016) \\
\hline Xianghe & Rural & $39.90,116.96$ & $\mathrm{PM}_{10}$ & AE31 & 2013.04-2015.03 & 5 & (Ran et al., 2016) \\
\hline Beijing & Urban-rural & $40.04,116.41$ & $\mathrm{PM}_{2.5}$ & AE31 & 2014 & $4.4 \pm 3.7$ & (Ji et al., 2017) \\
\hline Beijing & Urban & $39.98,116.30$ & $\mathrm{NP}$ & AE33 & $2015.12-2016.02$ & $5.31 \pm 6.26$ & (Liu et al., 2018) \\
\hline \multicolumn{8}{|l|}{ Tibetan } \\
\hline Lulang & Remote area & $29.46,94.44$ & & $\mathrm{SP} 2$ & 2015.09-10 & $0.31 \pm 0.55$ & (Wang et al., 2018) \\
\hline Qinghai Lake & Remote area & $36.98,99.88$ & & $\mathrm{SP} 2$ & $2012.11 .16-27$ & $0.16 \pm 0.19$ & (Wang et al., 2015) \\
\hline Nam Co & Remote area & $30.77,90.99$ & $\mathrm{PM}_{1.0}$ & TOR & $2012.09-12$ & 0.09 & (Wan et al., 2015) \\
\hline Ranwu & Remote area & $29.32,96.96$ & $\mathrm{NP}$ & AE31 & $2012.11-2013.02$ & 0.41 & (Wang et al., 2016) \\
\hline Beiluhe & Remote area & $34.85,92.94$ & NP & AE31 & 2012.11-2013.02 & 0.2 & \\
\hline Muztagh Ata & Remote area & $38.28,75.02$ & NP & AE16 & 2009.09 & $0.16 \pm 0.19$ & (Zhu et al., 2016) \\
\hline \multicolumn{8}{|l|}{ National wide } \\
\hline Chengdu & Urban & $30.65,104.04$ & $\mathrm{PM}_{10}$ & TOR & 2006-07 Annual & $10.8 \pm 5.52$ & (Zhang et al., 2012) \\
\hline Dalian & Urban & $38.90,121.63$ & $\mathrm{PM}_{10}$ & TOR & 2006-07 Annual & $5.28 \pm 2.53$ & \\
\hline Dunhuang & Rural & $40.15,94.68$ & $\mathrm{PM}_{10}$ & TOR & 2006-07 Annual & $4.09 \pm 2.33$ & \\
\hline Gaolanshan & Rural & $36.00,105.85$ & $\mathrm{PM}_{10}$ & TOR & 2006-07 Annual & $3.79 \pm 2.01$ & \\
\hline Gucheng & Rural & $39.13,115.8$ & $\mathrm{PM}_{10}$ & TOR & 2006-07 Annual & $10.6 \pm 4.60$ & \\
\hline Jinsha & Rural & $39.63,114.2$ & $\mathrm{PM}_{10}$ & TOR & 2006-07 Annual & $2.98 \pm 1.21$ & \\
\hline Lhasa & Urban & $29.67,91.13$ & $\mathrm{PM}_{10}$ & TOR & 2006-07 Annual & $3.87 \pm 2.20$ & \\
\hline LinAn & Rural & $31.30,119.73$ & $\mathrm{PM}_{10}$ & TOR & 2006-07 Annual & $4.24 \pm 1.89$ & \\
\hline Longfengshan & Rural & $44.73,127.60$ & $\mathrm{PM}_{10}$ & TOR & 2006-07 Annual & $2.25 \pm 1.04$ & \\
\hline
\end{tabular}




\begin{tabular}{|c|c|c|c|c|c|c|c|}
\hline Nanning & Urban & $22.82,108.35$ & $\mathrm{PM}_{10}$ & TOR & 2006-07 Annual & $3.84 \pm 1.79$ & \\
\hline Panyu & Urban & $22.93,113.32$ & $\mathrm{PM}_{10}$ & TOR & 2006-07 Annual & $7.54 \pm 3.57$ & \\
\hline Taiyangshan & Rural & $29.17,111.71$ & $\mathrm{PM}_{10}$ & TOR & 2006-07 Annual & $2.61 \pm 1.15$ & \\
\hline Zhengzhou & Urban & $34.78,113.68$ & $\mathrm{PM}_{10}$ & TOR & 2006-07 Annual & $9.43 \pm 3.50$ & \\
\hline Xi'an & Urban & $34.43,108.97$ & $\mathrm{PM}_{10}$ & TOR & 2006-07 Annual & $12.1 \pm 4.62$ & \\
\hline Xi'an & Urban & $34.22,109.00$ & $\mathrm{PM}_{10}$ & SP2 & 2012.12-2013.01 & $8.8 \pm 3.7$ & (Wang et al., 2014b) \\
\hline Lanzhou & Rural & $35.57,104.08$ & $\mathrm{PM}_{2.5}$ & AE31 & 2007.01-2009.08 & 1.57 & (Zhang et al., 2011) \\
\hline Shenyang & Urban & $41.77,123.50$ & $\mathrm{NP}$ & AE31 & $2008.03-2009.02$ & 6.14 & (Wang et al., 2011) \\
\hline Dalian & Urban & $38.90,121.63$ & NP & AE31 & $2008.03-2009.02$ & 3.18 & \\
\hline Anshan & Urban & $41.08,123.00$ & NP & AE31 & $2008.03-2009.02$ & 5.3 & \\
\hline Fushun & Urban & $41.88,123.95$ & NP & AE31 & 2008.03-2009.02 & 4.16 & \\
\hline Benxi & Urban & $41.32,123.78$ & NP & $\mathrm{AE} 31$ & 2008.03-2009.02 & 6.78 & \\
\hline Changchun & Urban & $43.90,125.22$ & NP & AE31 & 2007.10-2008.01 & 15.6 & (Gao et al., 2009) \\
\hline \multicolumn{8}{|l|}{ Worldwide } \\
\hline \multicolumn{8}{|l|}{ Finland } \\
\hline Hyytiälä, & Forest & & $\mathrm{PM}_{10}$ & AE31 & 2004.12-2008.12 & $0.32 \pm 0.34$ & (Hyvärinen et al., 2011) \\
\hline Puijo & Finland & & $\mathrm{PM}_{2.5}$ & MAAP & 2006.08-2008.12 & $0.23 \pm 0.27$ & \\
\hline Utö & & & $\mathrm{PM}_{2.5}$ & AE31 & $2007.01-2008.12$ & $0.25 \pm 0.33$ & \\
\hline Virolahti & & & $\mathrm{PM}_{2.5}$ & AE31 & $2006.08-2008.12$ & $0.42 \pm 0.45$ & \\
\hline Pallastunturi & & & $\mathrm{PM}_{10}$ & MAAP & 2007.09-2008.12 & $0.06 \pm 0.13$ & \\
\hline \multicolumn{8}{|l|}{ Ontario } \\
\hline HWY401 & Near road & $43.71,79.54$ & $\mathrm{PM}_{2.5}$ & AE33 & $2015.06-2016.05$ & 1.74 & (Healy et al., 2017) \\
\hline Etobicoke & Near road & $43.61,79.52$ & $\mathrm{PM}_{2.5}$ & AE33 & $2015.06-2016.05$ & 0.95 & \\
\hline College St. G & Near road & $43.65,79.39$ & $\mathrm{PM}_{2.5}$ & AE33 & $2015.06-2016.05$ & 0.84 & \\
\hline College St. R & Near road & $43.65,79.39$ & $\mathrm{PM}_{2.5}$ & AE33 & $2015.06-2016.05$ & 0.59 & \\
\hline Scarborough & Near road & 43.74 .9 .27 & $\mathrm{PM}_{2.5}$ & AE22 & $2015.06-2016.05$ & 0.69 & \\
\hline Hamilton DTN & Near road & $43.25,79.86$ & $\mathrm{PM}_{2.5}$ & $\mathrm{AE} 22$ & $2015.06-2016.05$ & 0.61 & \\
\hline Windsor DTN & Near road & $42.31,83.04$ & $\mathrm{PM}_{2.5}$ & AE22 & $2015.06-2016.05$ & 0.55 & \\
\hline Windsor W & Residential & $42.29,83.07$ & $\mathrm{PM}_{2.5}$ & AE33 & $2015.06-2016.05$ & 0.72 & \\
\hline Hanlan's Point & Background & $43.61,79.38$ & $\mathrm{PM}_{2.5}$ & AE33 & 2015.06-2016.05 & 0.51 & \\
\hline France & & & & & & & (Petit et al., 2017) \\
\hline SIRTA & Semi-urban & $48.71,2.15$ & & AE33 & & 0.8 & \\
\hline Metz & Urban & $49.11,6.22$ & & AE33 & & 2.3 & \\
\hline Lyon & Urban & $45.76,4.85$ & & AE33 & & 1.7 & \\
\hline South Africa & & & & & & & (Chiloane et al., 2017) \\
\hline Enlandsfontein & Top of hill & $26.25(\mathrm{~s}), 29.42$ & & MAAP & 2009.01-2011.05 & 0.8 & \\
\hline Marikana & Village & $25.70(\mathrm{~s}), 27.38$ & & MAAP & $2008.01-1010.05$ & 1.2 & \\
\hline Amersfoort & & $27.07(\mathrm{~s}), 29.87$ & & MAAP & 2010.01-2012.05 & 1.1 & \\
\hline
\end{tabular}

TOR: Thermal/optical reflectance carbon analyzer;

SP2: single particle soot photometer;

MAAP: multi-angle absorption photometer;

Anthalometer, Magee Scientific, Mode 
Table S2 Reported absorption coefficients of BC in China

\begin{tabular}{|c|c|c|c|c|c|}
\hline Stations & Locations $\left({ }^{\circ} \mathrm{N}\right),\left({ }^{\circ} \mathrm{E}\right)$ & Instrument & Sampling period & $\sigma_{\mathrm{ap}}(\lambda \mathrm{nm})$ & References \\
\hline Hongan & $31.24,114.58$ & AE33 & 2018.01 & $86.0(550)$ & This study \\
\hline Luohe & $33.57,114.05$ & AE33 & 2018.01 & $132(550)$ & \\
\hline Wuhan & $30.53,114.39$ & AE31 & 2018.01 & $60.6(550)$ & \\
\hline \multicolumn{6}{|l|}{ YRD } \\
\hline \multirow[t]{3}{*}{ Nanjing } & $32.10,119.90$ & AE31 & $2013.06-2015.05$ & $26.1(520)$ & (Shen et al., 2017) \\
\hline & $32.20,118.70$ & PASS & $2011.03-04$ & $28.1(532)$ & (Yu et al., 2016) \\
\hline & $32.05,118.78$ & AE31 & $2014.03-2016.02$ & $29.6(520)$ & (Zhuang et al., 2017) \\
\hline \multirow[t]{3}{*}{ Shanghai } & $31.30,121.48$ & AE31 & $2010.12-2011.03$ & $66(532)$ & (Xu et al., 2012) \\
\hline & $31.30,121.48$ & AE31 & $2010.12-2012.10$ & $38(532)$ & (Cheng et al., 2015) \\
\hline & $40.04 \mathrm{~N}, 116.41$ & AE31 & 2014 & $38.7(880)$ & (Ji et al., 2017) \\
\hline Shouxian & $32.56,116.78$ & PSAP & $2008.05-12$ & $29.4(550)$ & (Fan et al., 2010) \\
\hline Lin'an & $30.28,119.75$ & PSAP & $1999.10-12$ & $23(565)$ & (Xu et al., 2002) \\
\hline \multicolumn{6}{|l|}{ PRD } \\
\hline \multirow[t]{2}{*}{ Guangzhou } & $23.00,113.35$ & & $2004-2007$ & $82(532)$ & (Wu et al., 2009) \\
\hline & $23.92,113.12$ & & 2006.07 & $42.5(532)$ & (Garland et al., 2008) \\
\hline Hong Kong & $22.22,114.25$ & AE31 & 2012-2015 & $8.30(550)$ & (Wang et al., 2017b) \\
\hline Panyu & $23.00,113.35$ & AE31 & $2014.02-03$ & $45.7(550)$ & (Tan et al., 2016) \\
\hline Xinken & $22.60,113.60$ & MAAP & $2004.10-11$ & $37(550)$ & (Cheng et al., 2008) \\
\hline \multicolumn{6}{|l|}{ NCP } \\
\hline \multirow[t]{2}{*}{ Beijing } & $39.85,116.52$ & PAS & 2006.08 & $51.8(532)$ & (Garland et al., 2009) \\
\hline & $39.98,116.32$ & AE16 & $2005.01-2006.12$ & $56(525)$ & (He et al., 2009) \\
\hline Tongyu & $44.56,122.92$ & AE31 & 2010.03 & $7.61(520)$ & $(\mathrm{Wu}, 2012)$ \\
\hline Wuqing & $39.38,117.02$ & MAAP & $2009.03-05$ & $19.1(550)$ & (Ma et al., 2011) \\
\hline Xianghe & $39.75,116.96$ & PSAP & 2005.05 & $65.0(550)$ & (Li et al., 2007) \\
\hline \multicolumn{6}{|l|}{ Tibetan } \\
\hline Lhasa & $29.60,91.10$ & AE33 & $2015.09-11$ & $53(370)$ & (Zhu et al., 2017) \\
\hline Lulang & $29.76,94.73$ & & & $15(370)$ & \\
\hline Qinghai Lake & $36.98,99.88$ & SP2 & $2012.11 .16-27$ & 2.1 & (Wang et al., 2015) \\
\hline \multicolumn{6}{|l|}{ National wide } \\
\hline Chengdu & $30.62,104.07$ & AE31 & $2015.01-02$ & 60.2 & (Wang et al., 2017a) \\
\hline Chongqin & $29.62,106.50$ & & $2015.01-02$ & 66.6 & \\
\hline Xiamen & $24.60,118.05$ & PAX & $2013.11-2014.01$ & 22.4 & (Deng et al., 2016) \\
\hline Xi'an & $34.23,108.88$ & PAX & $2012.8-10$ & $31(532)$ & (Zhu et al., 2015) \\
\hline Jinan & $36.67,117.05$ & AE21 & $2013.12-2014.2$ & $63(532)$ & (Yan et al., 2017) \\
\hline Tuoji & $38.19,120.74$ & AE21 & $2014.12-2015.01$ & $8(532)$ & \\
\hline Yulin & $38.33,109.72$ & PSAP & $2001.03-05$ & 6 & $(\mathrm{Xu}, 2004)$ \\
\hline
\end{tabular}

Photoacoustic Extinctiometers, Boulder, PAX

Absorption Photometer, Radiance Research, PSAP 
Table S3 Differences of meteorological conditions (temperature (T), relative humidity $(\mathrm{RH})$, pressure (P), visibility (Vis), and boundary layer height (BLH)) at the five observation sites.

\begin{tabular}{|c|c|c|c|c|c|}
\hline & & $\mathrm{HA}$ & $\mathrm{LH}$ & SX & $\mathrm{WH}$ \\
\hline \multirow[t]{4}{*}{$\mathrm{T}$} & LH & & & & \\
\hline & SX & & & & \\
\hline & WH & & & & \\
\hline & $X Y$ & & & & \\
\hline \multirow[t]{4}{*}{ RH } & LH & & & & \\
\hline & SX & & & & \\
\hline & WH & & & & \\
\hline & $X Y$ & & & & \\
\hline \multirow[t]{4}{*}{$\mathrm{P}$} & LH & & & & \\
\hline & SX & & & & \\
\hline & WH & & & & \\
\hline & $X Y$ & & & & \\
\hline \multirow[t]{4}{*}{ Vis } & LH & & & & \\
\hline & SX & & & & \\
\hline & WH & & & & \\
\hline & $X Y$ & & & & \\
\hline \multirow[t]{4}{*}{ BLH } & LH & & & & \\
\hline & SX & & & & \\
\hline & WH & & & & \\
\hline & $X Y$ & & & & \\
\hline
\end{tabular}

Pink color means that the difference between the two variables was significant at 0.05 level; yellow color means that the difference between two variables was not significant. 
Table S4 Number of data for the different air quality in Figure 4.

\begin{tabular}{llllll}
\hline & HA & LH & SX & WH & XY \\
\hline Clean & 17424 & 7629 & 10500 & 1983 & 2315 \\
Light pollution & 7650 & 14303 & 9720 & 1632 & 13334 \\
Heavy pollution & $/$ & 2313 & $/$ & $/$ & 2856 \\
\hline
\end{tabular}

/: no heavy pollution episode existed. 


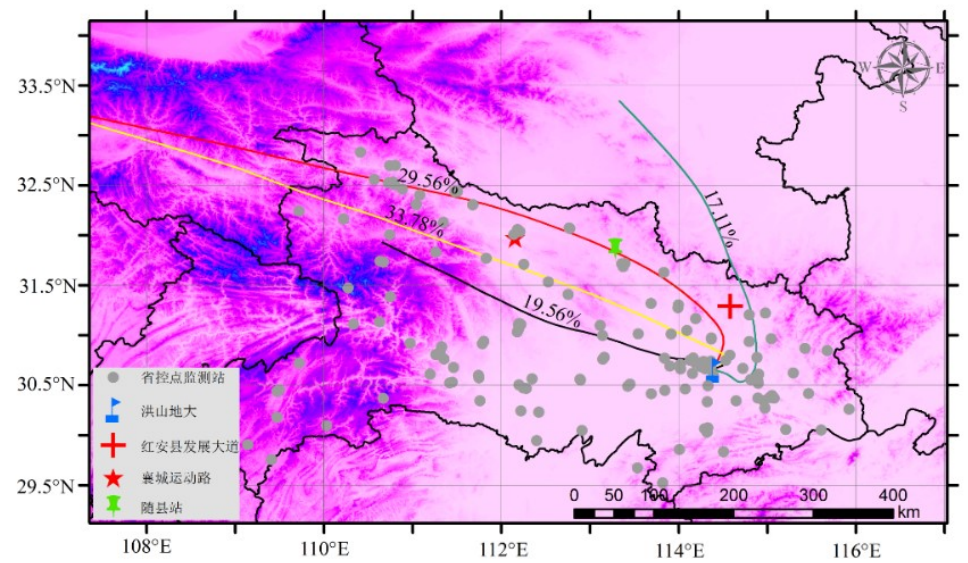

Figure S1 Cluster analysis results of air masses transported to Wuhan in January 2017. 


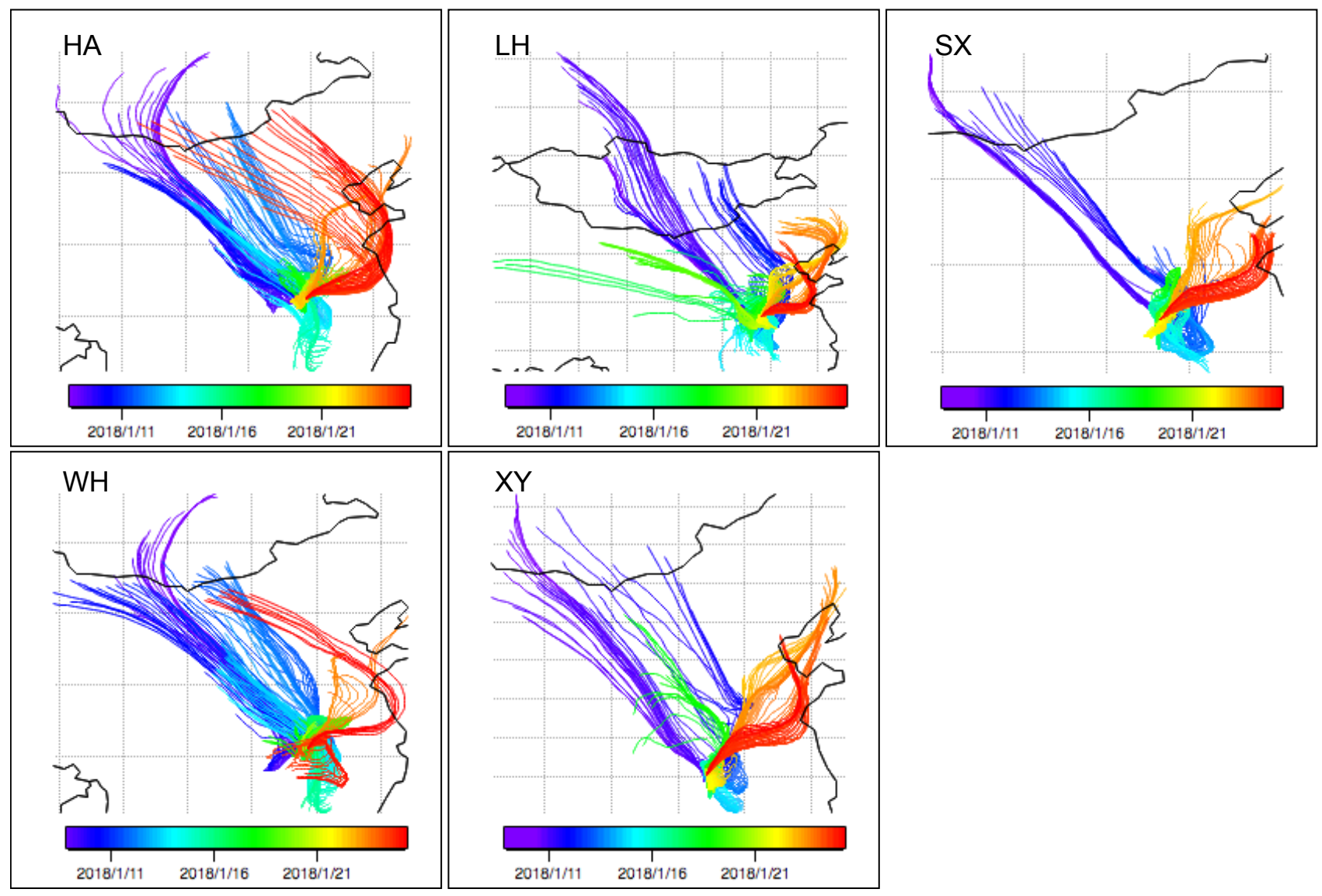

Figure S2 Hourly trajectories reaching at the five sites during the observation period. 

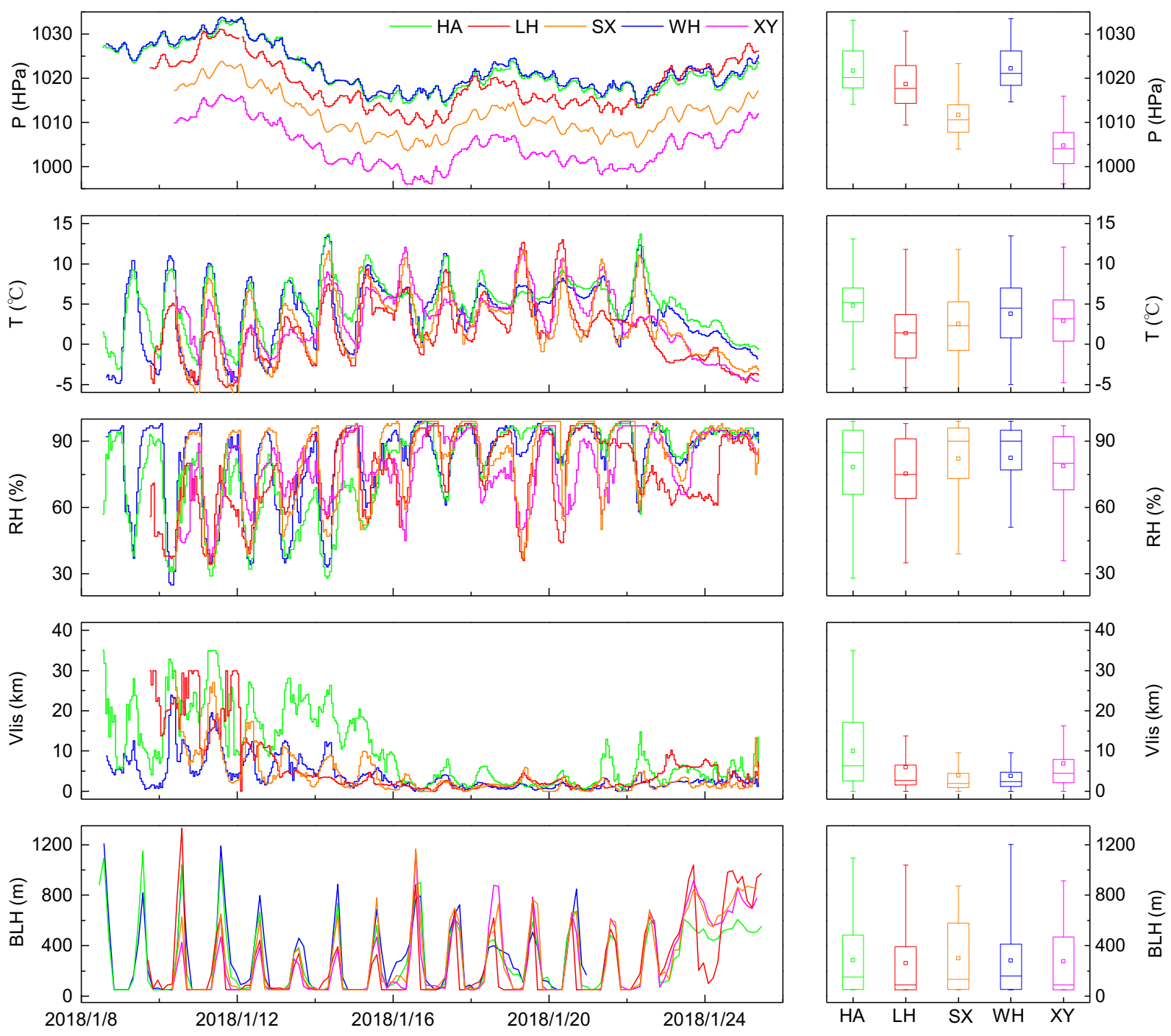

Figure S3 Time series and box plots of meteorological parameters including pressure $(\mathrm{P})$, temperature $(\mathrm{T})$, relative humidity (RH), visibility (Vis), and boundary layer height (BLH) at the five sites. 

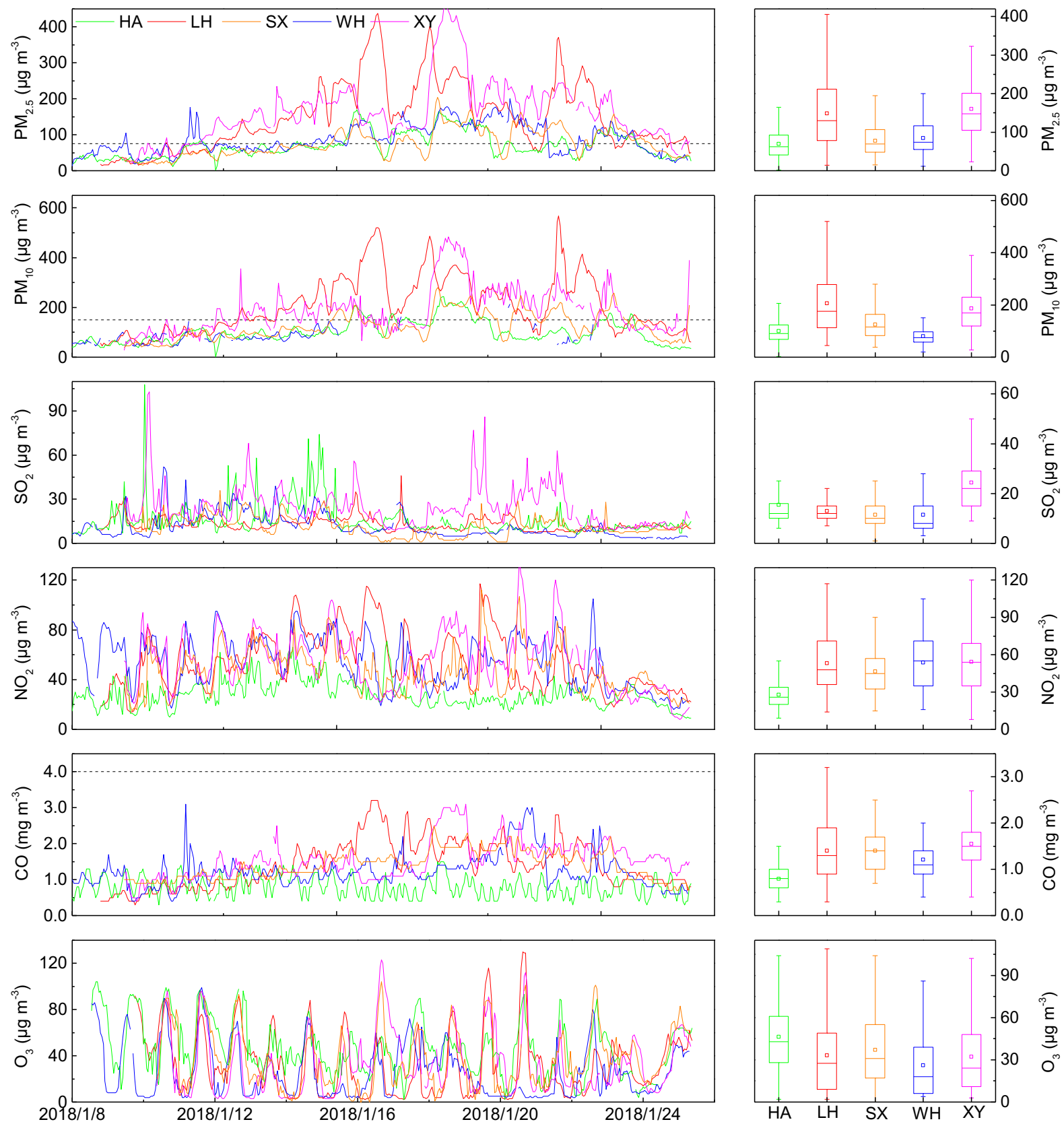

Figure $\mathbf{S} 4$ Time series and box plots of the hourly averaged $\mathrm{PM}_{2.5}, \mathrm{PM}_{10}, \mathrm{SO}_{2}, \mathrm{NO}_{2}, \mathrm{CO}$, and $\mathrm{O}_{3}$ concentrations at the five sites for the observation period (2018/1/8 2018/1/25). The dash lines are the corresponding daily secondary standards of the ambient air quality in China (GB3095-2012). The $\mathrm{SO}_{2}, \mathrm{CO}$ and $\mathrm{O}_{3}$ concentrations were lower than their secondary standards $\left(150 \mu \mathrm{g} \mathrm{m}^{-3}\right.$ for $\mathrm{SO}_{2}, 4 \mathrm{mg} \mathrm{m}^{-3}$ for $\mathrm{CO}$, and $160 \mu \mathrm{g} \mathrm{m}^{-3}$ for $\left.\mathrm{O}_{3}\right)$ during the entire periods. 


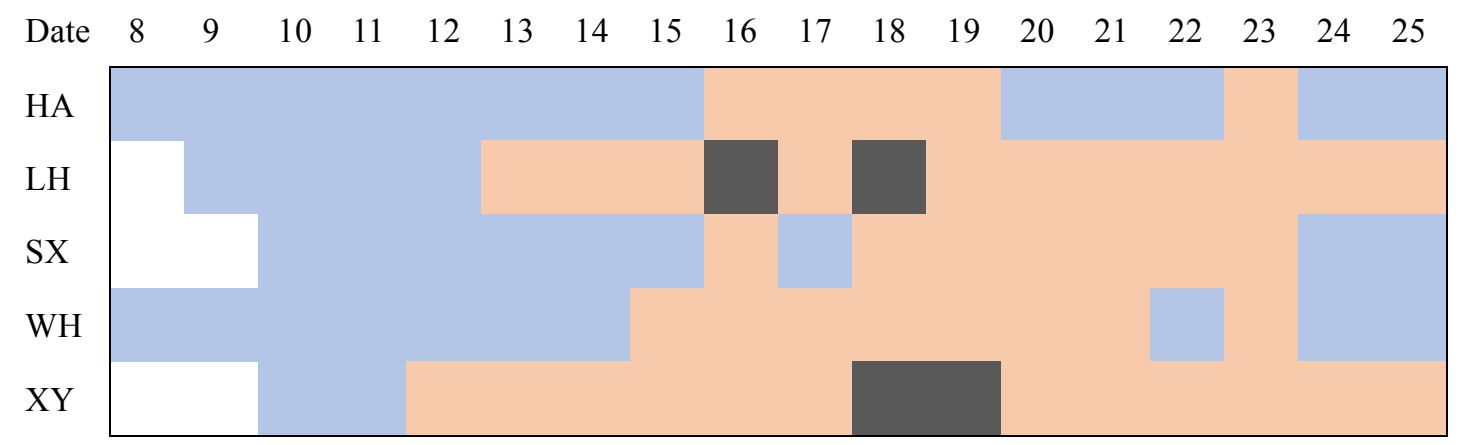

Figure S5 Daily air quality of each site during the observation periods. The blue, orange and dark stand for good $\left(\mathrm{PM}_{2.5}<\right.$ $\left.75 \mu \mathrm{g} \mathrm{m}^{-3}\right)$, light polluted $\left(75<\mathrm{PM}_{2.5}<250 \mu \mathrm{g} \mathrm{m}^{-3}\right)$ and heavily polluted $\left(\mathrm{PM}_{2.5}>250 \mu \mathrm{g} \mathrm{m}^{-3}\right)$ air quality, respectively. 

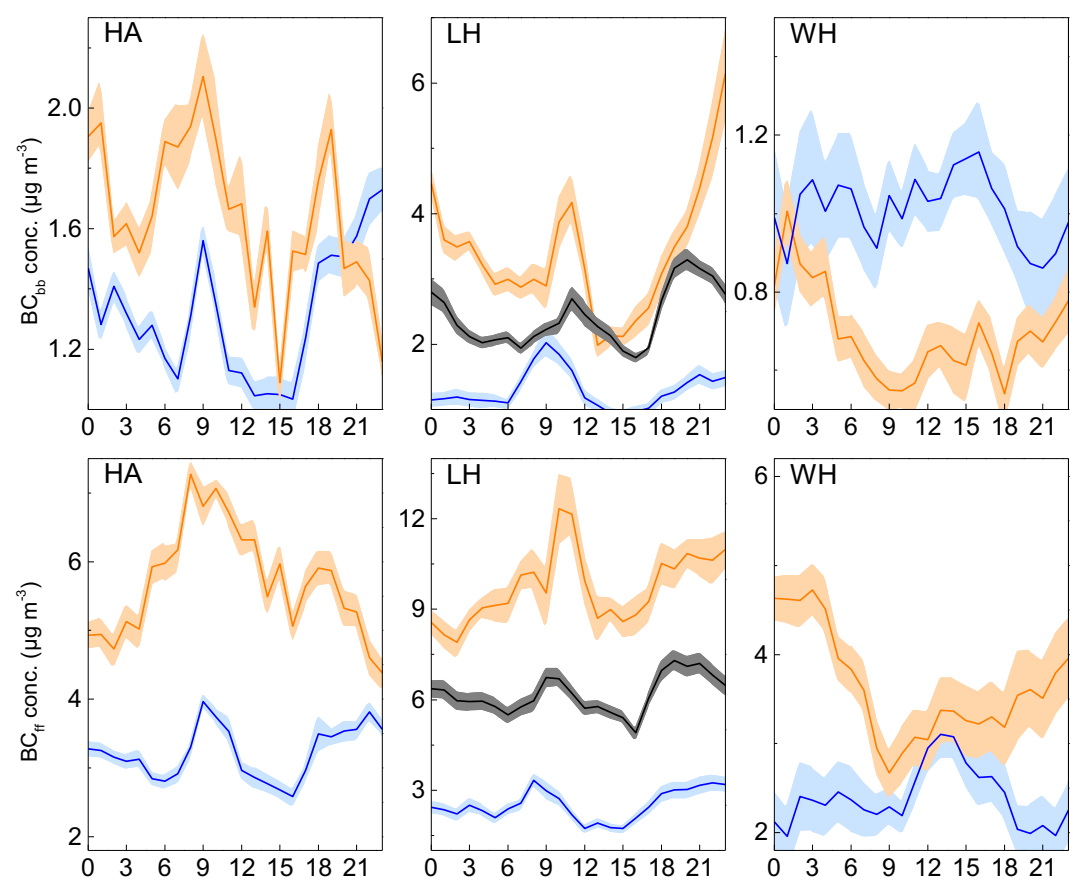

Figure S6 Diurnal variations of $\mathrm{BC}_{b b}$ (up panel) and $\mathrm{BC}_{f f}$ (bottom panel) at $\mathrm{HA}, \mathrm{LH}$ and WH under different air quality. 

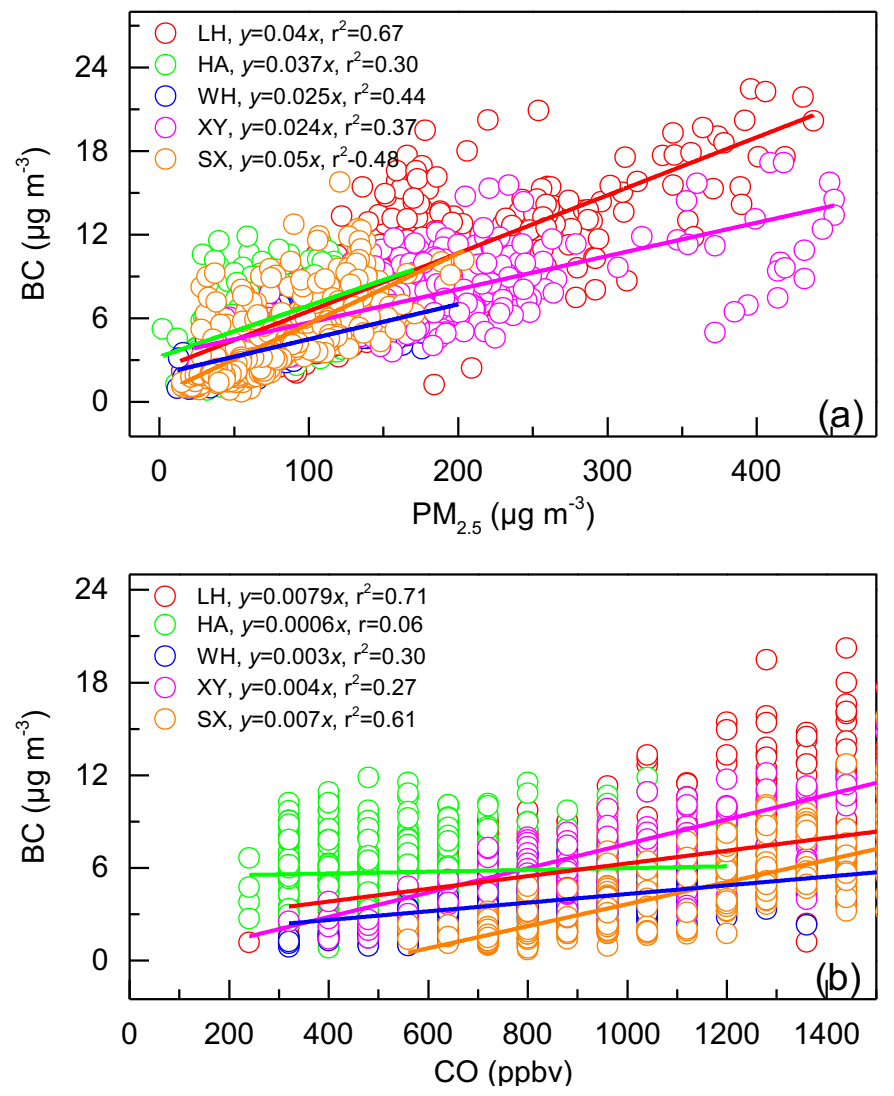

Figure S7 Scatter plots of $\mathrm{BC}$ vs $\mathrm{PM}_{2.5}$ (a) and $\mathrm{BC}$ vs $\mathrm{CO}(\mathrm{b})$. 


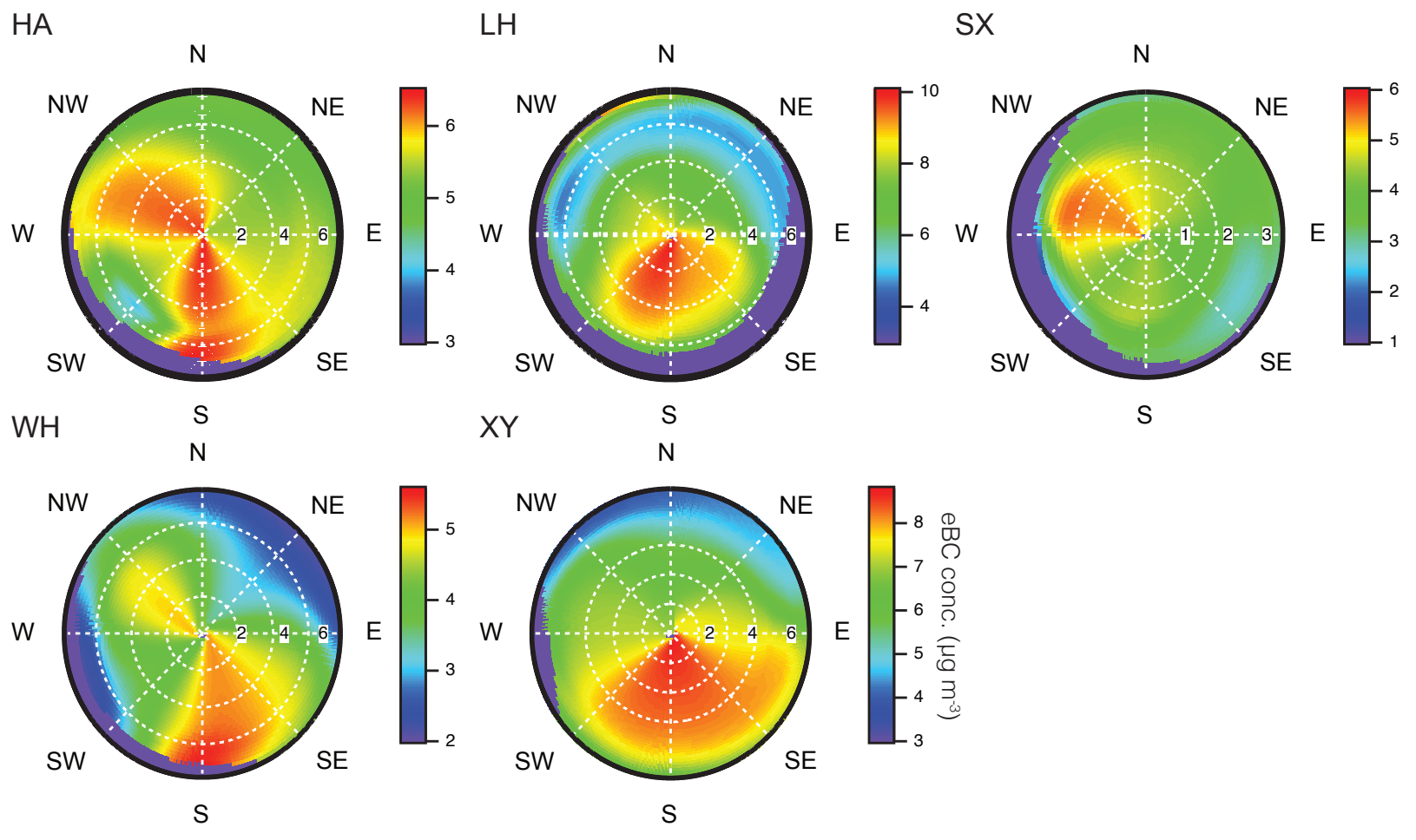

Figure S8 Nonparametric wind regression (NWR) plots of eBC at the five observation sites. 

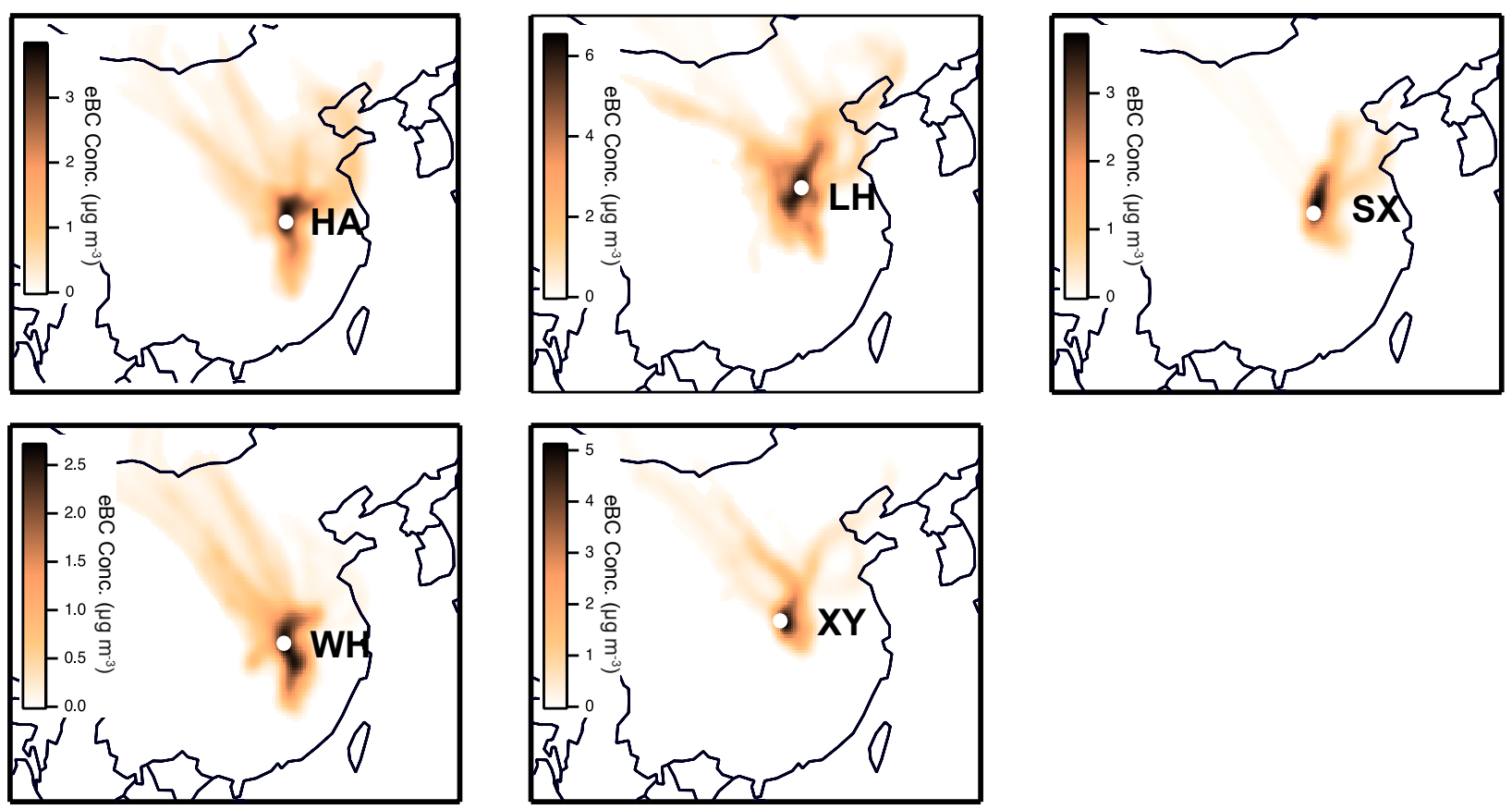

Figure S9 CWT results of $\mathrm{eBC}$ at the five sites during the observation period 


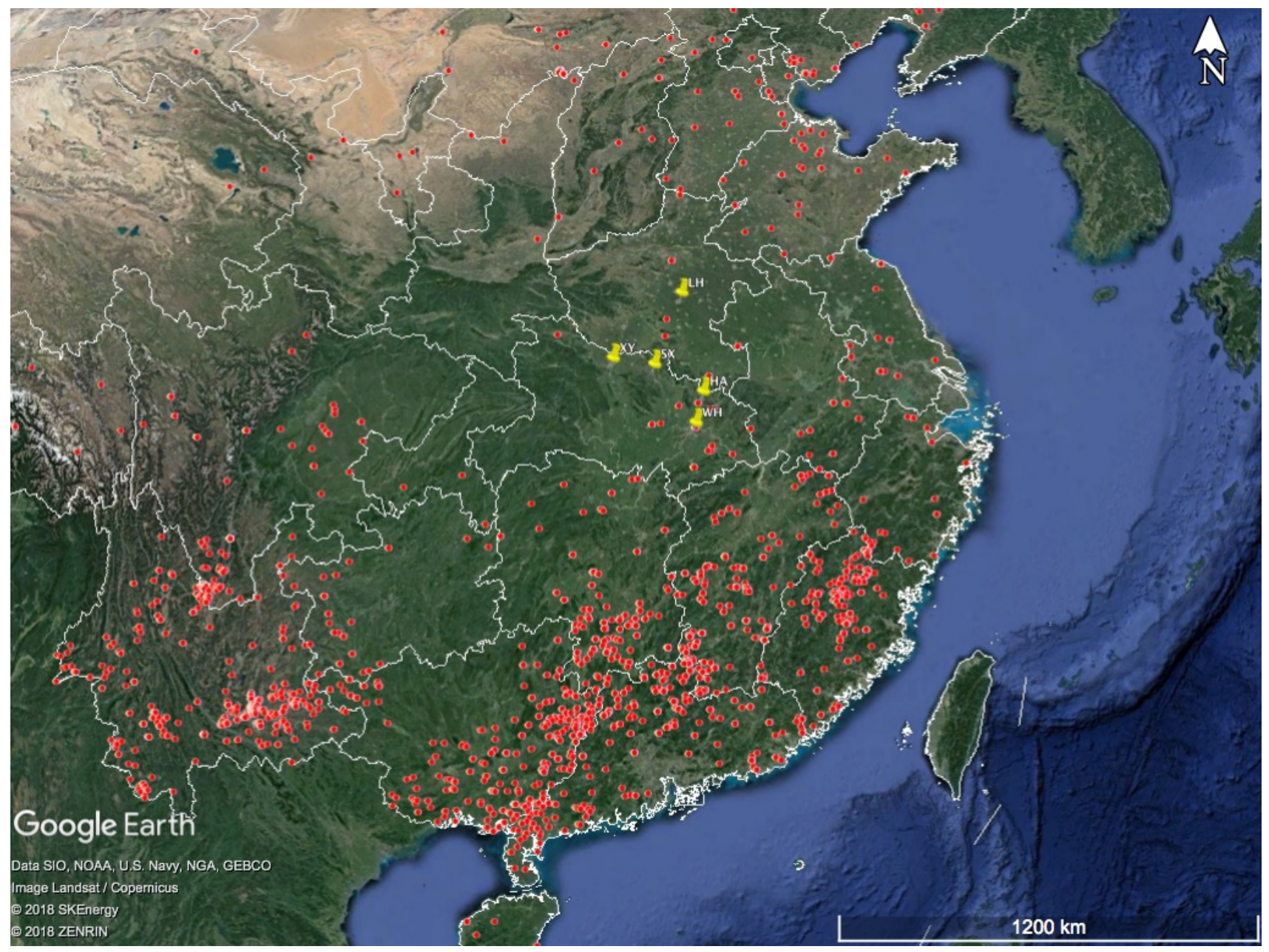

Figure S10 Locations of the fire spots downloaded from MODIS during the observation period (2018/1/08 2018/1/25). 


\section{Reference}

Cheng, T., Xu, C., Duan, J., Wang, Y., Leng, C., Tao, J., Che, H., He, Q., Wu, Y., Zhang, R., Li, X., Chen, J., Kong, L. and $\mathrm{Yu}, \mathrm{X}$.: Seasonal variation and difference of aerosol optical properties in columnar and surface atmospheres over Shanghai, Atmos. Environ., 123, 315-326, doi:10.1016/j.atmosenv.2015.05.029, 2015.

Cheng, Y. F., Wiedensohler, A., Eichler, H., Su, H., Gnauk, T., Brüggemann, E., Herrmann, H., Heintzenberg, J., Slanina, J. and Tuch, T.: Aerosol optical properties and related chemical apportionment at Xinken in Pearl River Delta of China, Atmos. Environ., 42(25), 6351-6372, doi:10.1016/j.atmosenv.2008.02.034, 2008.

Chiloane, K. E., Beukes, J. P., van Zyl, P. G., Maritz, P., Vakkari, V., Josipovic, M., Venter, A. D., Jaars, K., Tiitta, P., Kulmala, M., Wiedensohler, A., Liousse, C., Mkhatshwa, G. V., Ramandh, A. and Laakso, L.: Spatial, temporal and source contribution assessments of black carbon over the northern interior of South Africa, Atmos. Chem. Phys., 17(10), 6177-6196, doi:10.5194/acp-17-6177-2017, 2017.

Deng, J., Zhang, Y., Hong, Y., Xu, L., Chen, Y., Du, W. and Chen, J.: Optical properties of PM 2.5 and the impacts of chemical compositions in the coastal city Xiamen in China, Sci. Total Environ., 557-558, 665-675, doi:10.1016/j.scitotenv.2016.03.143, 2016.

Fan, X., Chen, H., Xia, X., Li, Z. and Cribb, M.: Aerosol optical properties from the Atmospheric Radiation Measurement Mobile Facility at Shouxian, China, J. Geophys. Res., 115, doi:10.1029/2010JD014650, 2010.

Gao, C., Zhang, R. and Su, L.: Characteristic analysis of atmospheric black carbon aerosols in Changchun in autumn and winter, Plateau Meteorol., 28(4), 803-807, 2009.

Garland, R. M., Yang, H., Schmid, O., Rose, D., Nowak, A., Achtert, P., Wiedensohler, A., Takegawa, N., Kita, K., Miyazaki, Y., Kondo, Y., Hu, M., Shao, M., Zeng, L. M., Zhang, Y. H., Andreae, M. O. and Pöschl, U.: Aerosol optical properties in a rural environment near the mega-city Guangzhou, China: implications for regional air pollution, radiative forcing and remote sensing,Atmos. Chem. Phys., 8(17), 5161-5186, doi:10.5194/acp-8-5161-2008, 2008.

Garland, R. M., Schmid, O., Nowak, A., Achtert, P., Wiedensohler, A., Gunthe, S. S., Takegawa, N., Kita, K., Kondo, Y., Hu, M., Shao, M., Zeng, L. M., Zhu, T., Andreae, M. O. and Pöschl, U.: Aerosol optical properties observed during Campaign of Air Quality Research in Beijing 2006 (CAREBeijing-2006): Characteristic differences between the inflow and outflow of Beijing city air, J. Geophys. Res., 114, doi:10.1029/2008JD010780, 2009.

He, X., Li, C. C., Lau, A. K. H., Deng, Z. Z., Mao, J. T., Wang, M. H. and Liu, X. Y.: An intensive study of aerosol optical properties in Beijing urban area, Atmos. Chem. Phys., 9(22), 8903-8915, doi:10.5194/acp-9-8903-2009, 2009.

Healy, R. M., Sofowote, U., Su, Y., Debosz, J., Noble, M., Jeong, C.H., Wang, J. M., Hilker, N., Evans, G. J., Doerksen, G., Jones, K. and Munoz, A.: Ambient measurements and source apportionment of fossil fuel and biomass burning black carbon in Ontario, Atmos. Environ., 161, 34-47, doi:10.1016/j.atmosenv.2017.04.034, 2017.

Huang, X.F., Sun, T.L., Zeng, L.W., Yu, G.H. and Luan, S.J.: Black carbon aerosol characterization in a coastal city in South China using a single particle soot photometer, Atmos. Environ., 51, 21-28, 
doi:10.1016/j.atmosenv.2012.01.056, 2012.

Huang, X.F., Xue, L., Tian, X.-D., Shao, W.-W., Sun, T.L., Gong, Z.H., Ju, W.W., Jiang, B., Hu, M. and He, L.Y.: Highly time-resolved carbonaceous aerosol characterization in Yangtze River Delta of China: Composition, mixing state and secondary formation, Atmos. Environ., 64, 200-207, doi:10.1016/j.atmosenv.2012.09.059, 2013.

Hyvärinen, A.P., Kolmonen, P., Kerminen, V.M., Virkkula, A., Leskinen, A., Komppula, M., Hatakka, J., Burkhart, J., Stohl, A., Aalto, P., Kulmala, M., Lehtinen, K. E. J., Viisanen, Y. and Lihavainen, H.: Aerosol black carbon at five background measurement sites over Finland, a gateway to the Arctic, Atmos. Environ., 45(24), 4042-4050, doi:10.1016/j.atmosenv.2011.04.026, 2011.

Ji, D., Li, L., Pang, B., Xue, P., Wang, L., Wu, Y., Zhang, H. and Wang, Y.: Characterization of black carbon in an urbanrural fringe area of Beijing, Environ. Pollut., 223, 524-534, doi:10.1016/j.envpol.2017.01.055, 2017.

Li, C., Marufu, L. T., Dickerson, R. R., Li, Z., Wen, T., Wang, Y., Wang, P., Chen, H. and Stehr, J. W.: In situ measurements of trace gases and aerosol optical properties at a rural site in northern China during East Asian Study of Tropospheric Aerosols: An International Regional Experiment 2005, J. Geophys. Res., 112(D22), doi:10.1029/2006JD007592, 2007.

Lin, C. Q., Liu, G., Lau, A. K. H., Li, Y., Li, C. C., Fung, J. C. H. and Lao, X. Q.: High-resolution satellite remote sensing of provincial PM 2.5 trends in China from 2001 to 2015, Atmos. Environ., 180, 110-116, doi:10.1016/j.atmosenv.2018.02.045, 2018.

Liu, Q., Ma, T., Olson, M. R., Liu, Y., Zhang, T., Wu, Y. and Schauer, J. J.: Temporal variations of black carbon during haze and non-haze days in Beijing, Sci. Rep., 6(1), doi:10.1038/srep33331, 2016.

Ma, N., Zhao, C. S., Nowak, A., Müller, T., Pfeifer, S., Cheng, Y. F., Deng, Z. Z., Liu, P. F., Xu, W. Y., Ran, L., Yan, P., Göbel, T., Hallbauer, E., Mildenberger, K., Henning, S., Yu, J., Chen, L. L., Zhou, X. J., Stratmann, F. and Wiedensohler, A.: Aerosol optical properties in the North China Plain during HaChi campaign: an in-situ optical closure study, Atmos. Chem. Phys., 11(12), 5959-5973, doi:10.5194/acp-11-5959-2011, 2011.

Petit, J.E., Amodeo, T., Meleux, F., Bessagnet, B., Menut, L., Grenier, D., Pellan, Y., Ockler, A., Rocq, B., Gros, V., Sciare, J. and Favez, O.: Characterising an intense PM pollution episode in March 2015 in France from multi-site approach and near real time data: Climatology, variabilities, geographical origins and model evaluation, Atmos. Environ., 155, 68-84, doi:10.1016/j.atmosenv.2017.02.012, 2017.

Shen, Y., Virkkula, A., Ding, A., Wang, J., Chi, X., Nie, W., Qi, X., Huang, X., Liu, Q., Zheng, L., Xu, Z., Petäjä, T., Aalto, P. P., Fu, C. and Kulmala, M.: Aerosol Optical Properties at SORPES in Nanjing, East China, Atmos. Chem. Phys. Discuss., 1-46, doi:10.5194/acp-2017-863, 2017.

Tan, H., Liu, L., Fan, S., Li, F., Yin, Y., Cai, M. and Chan, P. W.: Aerosol optical properties and mixing state of black carbon in the Pearl River Delta, China, Atmos. Environ., 131, 196-208, doi:10.1016/j.atmosenv.2016.02.003, 2016.

Tao, J., Zhang, L., Cao, J. and Zhang, R.: A review of current knowledge concerning PM 2.5 : chemical composition, 
aerosol optical properties and their relationships across China, Atmos. Chem. Phys., 17(15), 9485-9518, doi:10.5194/acp-17-9485-2017, 2017.

Wan, X., Kang, S., Wang, Y., Xin, J., Liu, B., Guo, Y., Wen, T., Zhang, G. and Cong, Z.: Size distribution of carbonaceous aerosols at a high-altitude site on the central Tibetan Plateau (Nam Co Station, 4730ma.s.1.), Atmos. Res., 153, 155164, doi:10.1016/j.atmosres.2014.08.008, 2015.

Wang, H., He, Q., Chen, Y. and Kang, Y.: Characterization of black carbon concentrations of haze with different intensities in Shanghai by a three-year field measurement, Atmos. Environ., 99, 536-545, doi:10.1016/j.atmosenv.2014.10.025, 2014a.

Wang, H., Shi, G., Tian, M., Zhang, L., Chen, Y., Yang, F. and Cao, X.: Aerosol optical properties and chemical composition apportionment in Sichuan Basin, China, Sci. Total Environ., 577, 245-257, doi:10.1016/j.scitotenv.2016.10.173, 2017a.

Wang, J., Virkkula, A., Gao, Y., Lee, S., Shen, Y., Chi, X., Nie, W., Liu, Q., Xu, Z., Huang, X., Wang, T., Cui, L. and Ding, A.: Observations of aerosol optical properties at a coastal site in Hong Kong, South China, Atmos. Chem. Phys., 17(4), 2653-2671, doi:10.5194/acp-17-2653-2017, $2017 \mathrm{~b}$.

Wang, M., Xu, B., Wang, N., Cao, J., Tie, X., Wang, H., Zhu, C. and Yang, W.: Two distinct patterns of seasonal variation of airborne black carbon over Tibetan Plateau, Sci. Total Environ., 573, 1041-1052, doi:10.1016/j.scitotenv.2016.08.184, 2016.

Wang, Q., Huang, R.J., Cao, J., Han, Y., Wang, G., Li, G., Wang, Y., Dai, W., Zhang, R. and Zhou, Y.: Mixing State of Black Carbon Aerosol in a Heavily Polluted Urban Area of China: Implications for Light Absorption Enhancement, Aerosol Sci. Technol., 48(7), 689-697, doi:10.1080/02786826.2014.917758, 2014b.

Wang, Q., Cao, J., Han, Y., Tian, J., Zhu, C., Zhang, Y., Zhang, N., Shen, Z., Ni, H., Zhao, S. and Wu, J.: Sources and physicochemical characteristics of black carbon aerosol from the southeastern Tibetan Plateau: internal mixing enhances light absorption, Atmos. Chem. Phys., 18(7), 4639-4656, doi:10.5194/acp-18-4639-2018, 2018.

Wang, Q. Y., Huang, R.-J., Cao, J. J., Tie, X. X., Ni, H. Y., Zhou, Y. Q., Han, Y. M., Hu, T. F., Zhu, C. S., Feng, T., Li, N. and Li, J. D.: Black carbon aerosol in winter northeastern Qinghai-Tibetan Plateau, China: the source, mixing state and optical property, Atmos. Chem. Phys., 15(22), 13059-13069, doi:10.5194/acp-15-13059-2015, 2015.

Wang, Y., Ma, Y., Lu, Z., Zhou, D., Liu, N., Zhang, Y. and Hong, Y.: In Situ Continuous Observation of Atmospheric Black Carbon Aerosol Mass Concentration in Liaoning Region, Res. Environ. Sci., 24(10), 1088-1096, 2011.

Weingartner, E., Saathoff, H., Schnaiter, M., Streit, N., Bitnar, B. and Baltensperger, U.: Absorption of light by soot particles: determination of the absorption coefficient by means of aethalometers, J. Aerosol Sci., 34(10), 1445-1463, doi:10.1016/S0021-8502(03)00359-8, 2003.

Wu, D., Mao, J., Deng, X., Tie, X., Zhang, Y., Zeng, L., Li, F., Tan, H., Bi, X., Huang, X., Chen, J. and Deng, T.: Black carbon aerosols and their radiative properties in the Pearl River Delta region, Sci. China Ser. Earth Sci., 52(8), 1152- 
1163, doi:10.1007/s11430-009-0115-y, 2009.

Wu, D., Wu, C., Liao, B., Chen, H., Wu, M., Li, F., Tan, H., Deng, T., Li, H., Jiang, D. and Yu, J. Z.: Black carbon over the South China Sea and in various continental locations in South China, Atmos. Chem. Phys., 13(24), 12257-12270, doi:10.5194/acp-13-12257-2013, 2013.

Wu, Y.: Aerosol Optical Properties Observed at a Semi-arid Rural Site in Northeastern China, Aerosol Air Qual. Res., doi:10.4209/aaqr.2011.11.0202, 2012.

Wu, Y., Zhang, R., Tian, P., Tao, J., Hsu, S.-C., Yan, P., Wang, Q., Cao, J., Zhang, X. and Xia, X.: Effect of ambient humidity on the light absorption amplification of black carbon in Beijing during January 2013, Atmos. Environ., 124, 217-223, doi:10.1016/j.atmosenv.2015.04.041, 2016.

$\mathrm{Xu}$, J.: Aerosol chemical, physical, and radiative characteristics near a desert source region of northwest China during ACE-Asia, J. Geophys. Res., 109(D19), doi:10.1029/2003JD004239, 2004.

Xu, J., Bergin, M. H., Yu, X., Liu, G., Zhao, J., Carrico, C. M. and Baumann, K.: Measurement of aerosol chemical, physical and radiative properties in the Yangtze delta region of China, Atmos. Environ., 36(2), 161-173, doi:10.1016/S1352-2310(01)00455-1, 2002.

Xu, J., Tao, J., Zhang, R., Cheng, T., Leng, C., Chen, J., Huang, G., Li, X. and Zhu, Z.: Measurements of surface aerosol optical properties in winter of Shanghai, Atmos. Res., 109-110, 25-35, doi:10.1016/j.atmosres.2012.02.008, 2012.

Yan, P., Tang, J., Huang, J., Mao, J. T., Zhou, X. J., Liu, Q., Wang, Z. F. and Zhou, H. G.: The measurement of aerosol optical properties at a rural site in Northern China, Atmos. Chem Phys, 14, 2008.

Yan, W., Yang, L., Chen, J., Wang, X., Wen, L., Zhao, T. and Wang, W.: Aerosol optical properties at urban and coastal sites in Shandong Province, Northern China, Atmos. Res., 188, 39-47, doi:10.1016/j.atmosres.2016.12.011, 2017.

Yu, X., Ma, J., Raghavendra Kumar, K., Zhu, B., An, J., He, J. and Li, M.: Measurement and analysis of surface aerosol optical properties over urban Nanjing in the Chinese Yangtze River Delta, Sci. Total Environ., 542, 277-291, doi:10.1016/j.scitotenv.2015.10.079, 2016.

Zhang, L., Zhang, L., Zhang, D., Zhao, S., Huang, J., Zhang, W. and Shi, J.: Property of black carbon concentration over outskirts of Lanzhou, Northwest China, China Environ. Sci., 31(8), 1248-1255, 2011.

Zhang, X. Y., Wang, Y. Q., Niu, T., Zhang, X. C., Gong, S. L., Zhang, Y. M. and Sun, J. Y.: Atmospheric aerosol compositions in China: spatial/temporal variability, chemical signature, regional haze distribution and comparisons with global aerosols,Atmos. Chem. Phys., 12(2), 779-799, doi:10.5194/acp-12-779-2012, 2012.

Zhu, C.S., Cao, J.J., Ho, K.F., Antony Chen, L.W., Huang, R.J., Wang, Y.C., Li, H., Shen, Z.X., Chow, J. C., Watson, J. G., Su, X., Wang, Q. and Xiao, S.: The optical properties of urban aerosol in northern China: A case study at Xi'an,Atmos. Res., 160, 59-67, doi:10.1016/j.atmosres.2015.03.008, 2015.

Zhu, C.S., Cao, J.J., Xu, B.Q., Huang, R.J., Wang, P., Ho, K.F., Shen, Z.X., Liu, S.X., Han, Y.M., Tie, X.X., Zhao, Z.Z. and Chen, L.W. A.: Black Carbon Aerosols at Mt. Muztagh Ata, a High-Altitude Location in the Western Tibetan 
Plateau, Aerosol Air Qual. Res., 16(3), 752-763, doi:10.4209/aaqr.2015.04.0255, 2016.

Zhu, C.S., Cao, J.J., Hu, T.F., Shen, Z.X., Tie, X.X., Huang, H., Wang, Q.Y., Huang, R.J., Zhao, Z.Z., Močnik, G. and Hansen, A. D. A.: Spectral dependence of aerosol light absorption at an urban and a remote site over the Tibetan Plateau, Sci. Total Environ., 590-591, 14-21, doi:10.1016/j.scitotenv.2017.03.057, 2017.

Zhuang, B., Wang, T., Liu, J., Li, S., Xie, M., Han, Y., Chen, P., Hu, Q., Yang, X., Fu, C. and Zhu, J.: The surface aerosol optical properties in the urban area of Nanjing, west Yangtze River Delta, China, Atmos. Chem. Phys., 17(2), 11431160, doi:10.5194/acp-17-1143-2017, 2017.

Zhuang, B. L., Wang, T. J., Liu, J., Li, S., Xie, M., Yang, X. Q., Fu, C. B., Sun, J. N., Yin, C. Q., Liao, J. B., Zhu, J. L. and Zhang, Y.: Continuous measurement of black carbon aerosol in urban Nanjing of Yangtze River Delta, China, Atmos. Environ., 89, 415-424, doi:10.1016/j.atmosenv.2014.02.052, 2014. 